\title{
Technology for a D-band/G-band ultra capacity layer
}

\author{
Claudio Paoloni ${ }^{1}$, Sebastian Boppel ${ }^{2}$, Viktor Krozer ${ }^{3}$, Trung Le $^{4}$, Rosa Letizia ${ }^{1}$, Ernesto Limiti ${ }^{5}$, François Magne ${ }^{6}$, \\ Marc Marilier ${ }^{7}$, Antonio Ramirez ${ }^{8}$, Borja Vidal ${ }^{9}$, Ralph Zimmerman ${ }^{4}$ \\ ${ }^{1}$ Engineering Department, Lancaster University, Lancaster, United Kingdom, LA1 4YW email: c.paoloni@ lancaster.ac.uk \\ ${ }^{2}$ Ferdinand-Braun-Institut, Leibniz-Institut für Höchstfrequenztechnik, Berlin, Germany \\ ${ }^{3}$ Goethe University of Frankfurt, Frankfurt 60323, Germany \\ ${ }^{4}$ HF Systems Engineering GmbH, Kassel 34123, Germany \\ ${ }^{5}$ University of Rome, Tor Vergata, Rome, Italy \\ ${ }^{6}$ When Ab, Paris, France \\ ${ }^{7}$ OMMIC S.A.S., Limeil Brevannes 94453, France \\ ${ }^{8}$ Fibernova Systems, Valencia 46022, Spain \\ ${ }^{9}$ Universitat Politècnica de València, Valencia 46022, Spain
}

\begin{abstract}
The bands above $100 \mathrm{GHz}$ offer outstanding potentiality for fixed wireless communications, matching the capacity requirements of future mobile networks backhaul in dense urban scenarios. However, millimeter wave components need a substantial technology advancement to enable such application. Fabrication challenges due to the short wavelength affecting the dimensions of the components, and the decrease of transmission power at the increase of the frequency are among the obstacles that limit the availability of working wireless systems above $100 \mathrm{GHz}$.

The Horizon 2020 project ULTRAWAVE is progressing in the enabling of the first ultracapacity layer for small cell backhaul by producing a network with Point to Multipoint sectors at D-band (141 -148.5 GHz) fed by Point to Point G-band (275 - 305 GHz) links.

The paper will describe the system specifications of the proposed ultracapacity layer and the millimeter wave technology in development in the ULTRAWAVE project.
\end{abstract}

Keywords-millimeter waves; backhaul; traveling wave tube; 5G; D-band;G-band; physical layer

\section{INTRODUCTION}

Millimeter waves above $90 \mathrm{GHz}$ are recognized as the portion of the spectrum to open for affordable and flexible backhaul and fiber-in-air at Gigabit per second $(\mathrm{Gb} / \mathrm{s})$ class. The recent prediction of five times increase of mobile data traffic by the 2024 [1, 2] describes how mobile wireless is becoming the predominant source of data. Small cell architectures are expected to be part of the future mobile network deployments in dense urban scenarios, where high capacity per $\mathrm{km}^{2}$ is required to meet subscriber demand. The lack of affordable high capacity backhaul systems to complement small cell deployments has prevented its proliferation among mobile network operators.

To provide capacity to a high number of cells per $\mathrm{km}^{2}$, operators need to deploy either a wide capillarity optical fiber network or to rely on millimeter wave wireless backhaul systems [3 - 8]. The former ensures high capacity but with a substantial

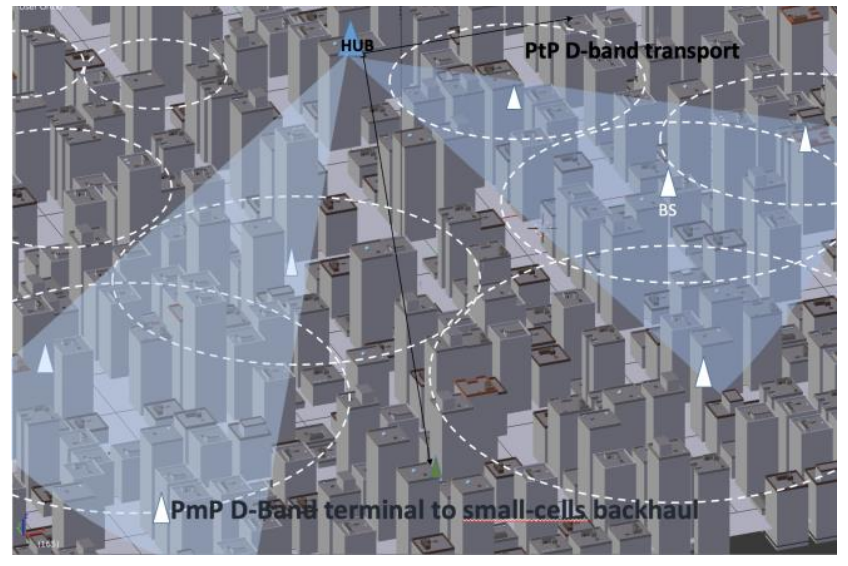

Fig. 1: Deployment scenario

deployment cost, while the latter has limited capacity due to spectrum and range limitations, and the complexity of deploying a high number of point to point links in a relatively small area.

In this paper, an approach to enable an ultracapacity wireless backhaul layer in untapped high frequency spectrum bands is presented. In the frame of the European Commission Horizon 2020 project ULTRAWAVE "Ultra-capacity wireless layer beyond $100 \mathrm{GHz}$ based on millimeter wave Traveling Wave Tubes" [9], the first backhaul architecture combining point to multipoint [10] sectorial distribution at D-band with point to point G-band transport links is designed (Fig. 1).

The availability of the ULTRAWAVE system will substantially impact on the economy of high capacity backhaul as alternative solution to fiber deployments [11 - 13]. All the components, subassemblies and assemblies at D-band and Gband present substantial design, fabrication and testing challenges and are at the state of the art and beyond.

The paper is structured as follows. Section II presents the ULTRAWAVE concept and provides the design specifications for the system, sub-assemblies and components. Section III 
focuses on the technology advancements required to fulfill ULTRAWAVE concept. Finally, Section IV offers some conclusions to the work presented in the paper.

\section{ULTRAWAVE CONCEPT}

The ULTRAWAVE architecture is an end-to-end backhaul network based on two millimeter wave systems above $100 \mathrm{GHz}$ : point to multipoint (PmP) wireless distribution networks at Dband, connected to the core fiber infrastructure by point to point (PtP) very high capacity links at G-band (Fig.2). Range and capacity of both systems are mainly supported by: a) Traveling Wave Tube (TWT) technology [12] that permits to achieve about $10-12$ Watt saturated power at D-band and 1-3 Watts at G-band, b) MMIC chip sets (Low-Noise Amplifiers, Power Amplifiers, and c) by the use of very wide bands $7.5 \mathrm{GHz}$ in D band and up to $30 \mathrm{GHz}$ in $\mathrm{G}$ band.

The PmP D-band will feed the backhaul of small cell sites at street level, while the G-band links will transport the aggregated capacity of the D-band sectors towards the operator's core network.

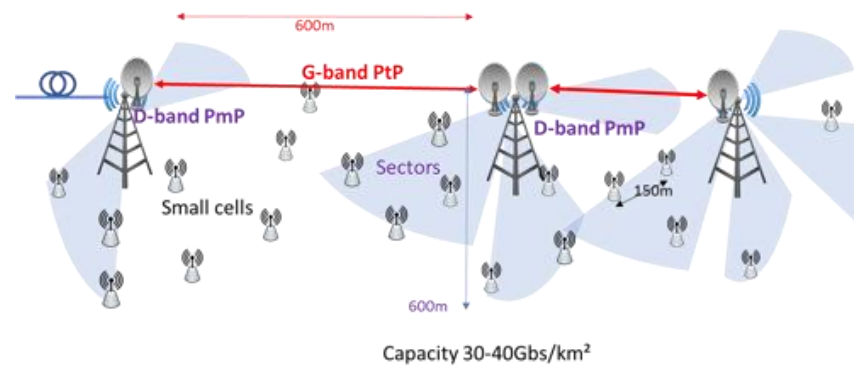

Fig. 2 ULTRAWAVE high capacity backhaul architecture.

The utilization of vacuum electronics, namely millimeterwave TWTs, provides the required transmission power for enabling D-band PmP with range of at least $500-600 \mathrm{~m}$, using a low directivity antenna in azimuth (sector) with min $23 \mathrm{dBi}$ (high directivity in elevation), and G-band PtP with a range of 600-700 $\mathrm{m}$ with high gain antenna (38 - $39 \mathrm{dBi})$. Range calculations consider rain condition in ITU zones $\mathrm{K}$ and $\mathrm{H}$ targeting around $99.99 \%$ availability, or $99.997 \%$ if ACM (Automatic Controlled Modulation) is adopted. Table I summarizes the targeted capacity and range for ULTRAWAVE systems.

The performance of the ULTRAWAVE high capacity layer aims at $100 \mathrm{~Gb} / \mathrm{s} / \mathrm{km}^{2}$, with aggregated data rates of $30 \mathrm{~Gb} / \mathrm{s}$, enabling a breakthrough in mobile internet access, providing unprecedented capacity for supporting the predicted internet growth.

\section{Table I}

ULTRAWAVE Architecture

\begin{tabular}{lllll}
\hline & Topology & Frequency & \multicolumn{1}{c}{ Capacity } & Range \\
$\begin{array}{l}\text { G-band } \\
\text { transport }\end{array}$ & $\begin{array}{l}\text { PtP } \\
\text { cascade, } \\
\text { loop or } \\
\text { mesh }\end{array}$ & $\begin{array}{l}\text { 30 Gb/s } \\
\text { aggregated } \\
\text { per link }\end{array}$ & $600 \mathrm{~m}$ \\
$\begin{array}{l}\text { D-band } \\
\text { distribution }\end{array}$ & $\begin{array}{l}\text { PmP } \\
\text { sectors }\end{array}$ & $141-148.5 \mathrm{GHz}$ & $\begin{array}{l}30 \mathrm{~Gb} / \mathrm{s} \\
\text { aggregated } \\
\text { per sector }\end{array}$ & $600 \mathrm{~m}$ \\
\hline
\end{tabular}

In the final stages of ULTRAWAVE project (2020), field trial measurements will be performed to demonstrate the above state of the art features utilizing the system developed within

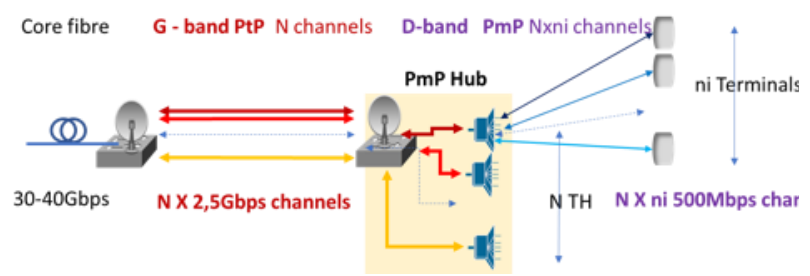

Fig. 3 System channelization and organization in ULTRAWAVE system.

ULTRAWAVE including the realized mm-wave TWTs as enabling device for $100 \mathrm{GHz}$ wireless access system.

\section{A. High capacity backhaul}

The ULTRAWAVE concept is based on the transmission of a frequency multiplex of TDD channels. In the D-band PmP, each of the channels of the multiplex is received and demodulated by a D-band terminal serving a small cell site. In the G-band PtP, each of multiplexed channels will aggregate the full capacity of a D-band sector. This architecture is shown in Fig.3. $N$ channels in the PtP link, with $N$ varying up to 12 , will feed capacity to $N$ different D-band hubs that will serve $n i$ terminals, with $n i$ varying up to 10 , depending on the capacity needs and ranges targeted on each D-band sector.

At the interconnection of both systems, the required capacity for the PmP hub is switched to the D-band system, while the rest of the multiplex in G-band can be transported to a further Dband hub in a cascaded PtP link.

Following the example in Fig. 3, a capacity of $30 \mathrm{~Gb} / \mathrm{s}$ could be distributed over twelve $2.5 \mathrm{~Gb} / \mathrm{s}$ channels in G-band, each of them allocated to a D-band sector where 5 to 10 terminals will share between $250 \mathrm{Mb} / \mathrm{s}$ and $500 \mathrm{Mb} / \mathrm{s}$ each.

The main benefit of Time Domain Division (TDD) is provided by the dynamic provision of DL/UL ratio, permitting to achieve the overall connectivity with maximum suppleness.

\section{B. Definition of link budget}

The link budget model for the ULTRAWAVE system takes into account numerous challenges in the system realization:

- Number of channels to multiplex.

- P1dB compression point of the amplifiers and associated back-off for linearity and PAPR.

- Output C/N: multiplexing of many channels is increasing the out-of-band channels depending on the shaping of the channel spectrum and the gap between channels.

- RF losses in the radio, particularly transitions to antennas and MMIC chain due to the high frequencies at the front-end.

- Antenna misalignment, both for PmP where terminals are not aligned in the center of the beam sector and PtP for possible face-to-face misalignments. 
Table II

\begin{tabular}{|l|r|r|}
\hline Europe ITU RAIN REGION K & G-band & D-band \\
\hline Modulation & QPSK $3 / 4$ & $16 Q A M 3 / 4$ \\
\hline Range (Km) & 2376.0 & 1056.0 \\
\hline Useful bit rate (Mbps)/Channel & 28512.0 & 10560.0 \\
\hline TOTAL CAPACITY/Link Mb/s & 12.8 & 16.6 \\
\hline C/N total (dB) & 3.5 & 2.5 \\
\hline margin Eb/NO & 290.0 & 145.0 \\
\hline Frequency (GHz) & 30.0 & 36.0 \\
\hline Amplifier 1dB-comp. point (dBm) & -1.5 & -1.0 \\
\hline Tx output losses (dB),Transisition & 17.7 & 25.0 \\
\hline Tx output power (dBm)/channel & 39.0 & 23.0 \\
\hline Tx Antenna Gain (dBi) & -0.5 & -3.0 \\
\hline Antenna pointing losses (dB) side & 56.2 & 43.0 \\
\hline Signal EIRP (dBmW)/cont & & \\
\hline Receiver Parameters: & & \\
\hline Total antenna gain (dBi) & 30.0 & 40.0 \\
\hline Antenna pointing losses (dB) & 39.9 & 99.99 \\
\hline Antenna + transition (dB) & -0.5 & 38.0 \\
\hline Down-converter noise figure (dB) & -2.0 & -0.5 \\
\hline Link data: & 8.5 & -1.5 \\
\hline Signal to interference ratio (dB) & & 5.0 \\
\hline System availability (\%) & & \\
\hline & & \\
\hline
\end{tabular}

- Minimum achievable noise figure NF.

- Gaseous absorption $\mathrm{H}_{2} \mathrm{O}$ (100\% humidity).

- Rain absorption for $99.99 \%$ availability in $\mathrm{K}$ rain area. It is to be noted that infrastructure operators would demand "five nine" (99.999\%) with ACM, therefore an extra $10 \mathrm{~dB}$ is required which could be obtained by reducing the MSC down to BPSK $1 / 2(+5 \mathrm{~dB})$ and reduce the channel width $(+6 \mathrm{~dB})$

- Roll-off which pertains to the filtering of the channel would give some carrier degradation.

- Phase noise: $-100 \mathrm{dBc} / \mathrm{Hz} @ 100 \mathrm{kHz}$ seems to be sufficient for $\mathrm{W}$ band and may degrade by $\sim 1 \mathrm{~dB}$ in $\mathrm{D}$ band. The phase noise at $300 \mathrm{GHz}$ has to be studied, because of a significant Eb/N0 degradation, among others due to increased multiplication factor when utilizing a synthesizer at Ka band for the local oscillator signal generation.

- Processing efficiency in considering sampling and quantization; it could lead to intermodulation and then Eb/N0 degradation.

The model employed in ULTRAWAVE computes both $\mathrm{C} / \mathrm{N}$ and $\mathrm{Eb} / \mathrm{N} 0$, noting that a correct $\mathrm{C} / \mathrm{N}$ obtained for the signal is not sufficient without a correct $\mathrm{Eb} / \mathrm{N} 0$ on the processing.

\section{Specifications}

Table II summarizes the main design parameters for the D-band PmP and G-band PtP layers for the rain zone K. The G-band link is assumed to work in QPSK, the D-band PmP link in
16QAM for link range of $600 \mathrm{~m}$. It is notable the relatively high transmission power above $30 \mathrm{dBm}$. This value is not achievable by solid state technology, hence the need of Travelling Wave Tube technology. The link budget includes the system losses and eventual misalignments of the antennas. The EIRP is computed to assure that the radiation level is below the hazard level.

\section{ULTRAWAVE TECHNOLOGY}

To satisfy the specifications in Table II, state of the art millimeter wave technology is needed. In the following the system and the components will be described.

\section{A. D-band point to multipoint backhaul}

The D-band $(141-148.5 \mathrm{GHz})$ is used to generate wide area sector with ultra capacity. Each area sector can have an aperture angle from $20^{\circ}$ to $60^{\circ}$ degree with a range up to $600 \mathrm{~m}$, equivalent to about $1000 \mathrm{~m}^{2}$ area. The number of channels allocated for each sector depends on the G-band link capacity and the capacity needed to support the traffic in the covered area.

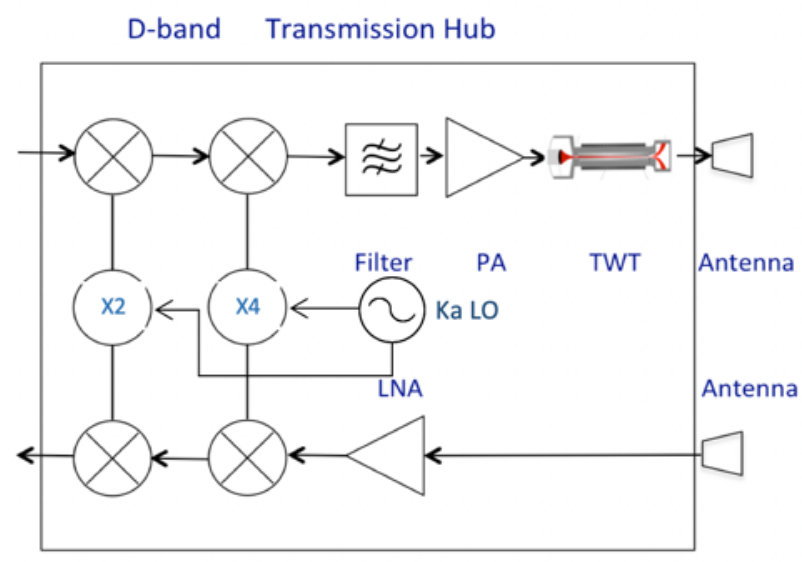

a)

D-band Terminal

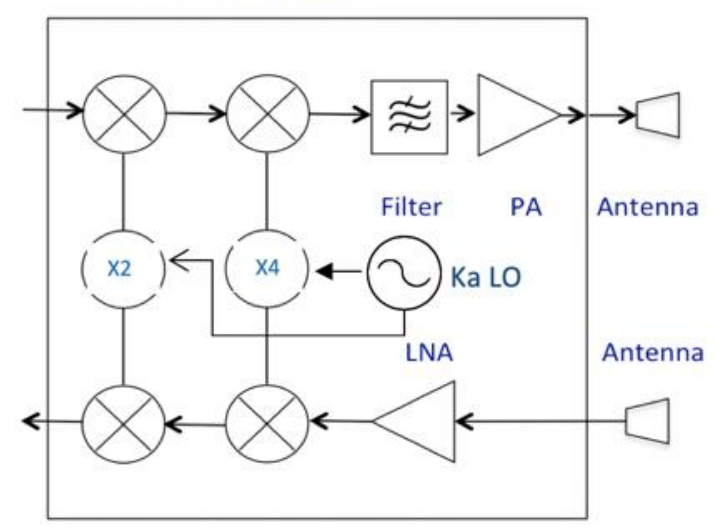

b)

Fig.4 Topology of the D-band (141 - 148.5 GHz) (a) transmission hub and (b) terminal. 


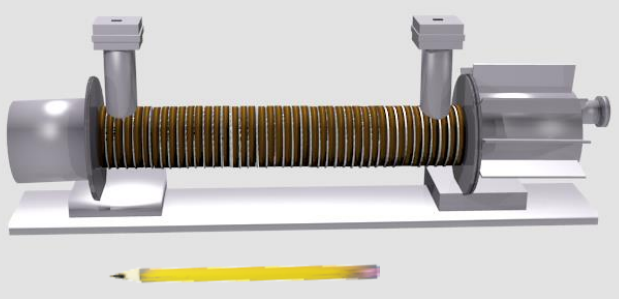

Fig.5 Artistic view of the D-band traveling wave tube

The full backhaul system is under development. It comprises a Transmission Hub (TH) outdoor unit and a number of Terminals.

The Transmission Hub schematic is shown in Fig. 4a. It includes the receiver and the transmitter. Two levels of IF for upconverter and downconverter are used in a super heterodyne architecture. A synthesizer with low phase noise and minimal spurious is adopted as local oscillator. The transmitter uses a high power traveling wave tube for achieving the range, connected to low gain horn antenna. The same antenna specifications are used for the receiver, in the range of $23 \mathrm{dBi}$. The low noise amplifier, frequency multiplier, and mixers are under realization in advanced GaAs technology.

The D-band terminal (schematic in Fig. 4b) is an all outdoor unit, to feed the backhaul network at street level for small cell.

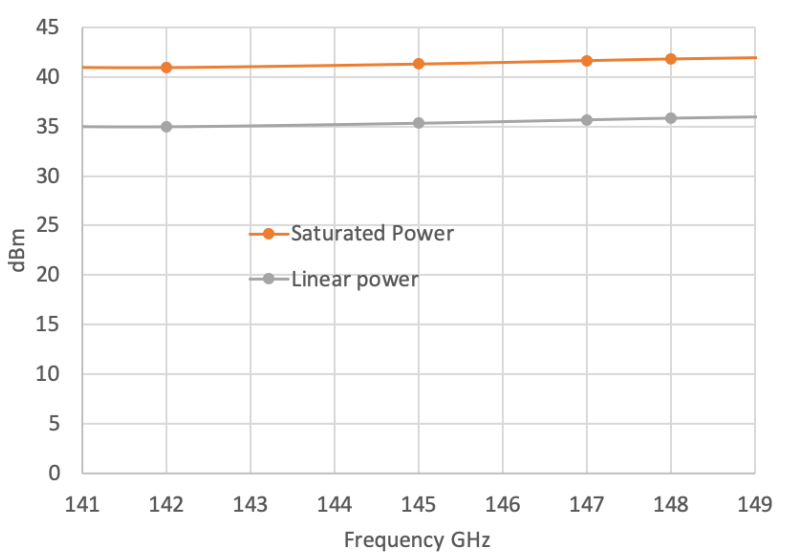

Fig. 6 Gain and output power of D-band TWT

It uses the same chipset of the Transmission Hub, with benefits on the final cost of the system. It uses two high gain directive antennas for the receiver and the transmitter. The same two level IF for the upconverter and downconverter is used.

The traveling wave tube (TWT) is in advanced fabrication. It will be one of the first TWTs worldwide at this frequency range. A gain of about $40 \mathrm{~dB}$ is achieved providing about $10 \mathrm{~W}$
$(40 \mathrm{dBm})$ saturated output power in order to provide about $3 \mathrm{~W}$ linear output power $(\mathrm{P} 1 \mathrm{~dB} \approx 35 \mathrm{dBm})$. The rendering of the TWT [14] is shown in Fig. 5. The power is shown in Fig.6 it is notable the wide frequency band and the performance fully satisfying the specifications [15].

As an example of the advanced technology used for the chipset, a MMIC power amplifier is realized using $800 \mathrm{~nm} \mathrm{InP}$ DHBT with three stages and 7 transistors (Fig. 7). The first measurements of the MMIC power amplifier shows about 16 $\mathrm{dBm}$ saturated power.

\section{B. G-Band Point to Point connecting link}

The G-band front end is the most advanced and challenging task of the project. A photonic transmitter to provide data rate of tens of $\mathrm{Gb} / \mathrm{s}$ is powered by a novel TWT with $30 \mathrm{GHz}$ bandwidth, driven by an InP MMIC power amplifier (Schematic in Fig. 8a). The photonic transmitter is shown in Fig. 8b. The Gband transmitter works on point to point due to the high attenuation and the limited power that the amplifiers, both MMIC and TWT can provide. The use of a relatively high

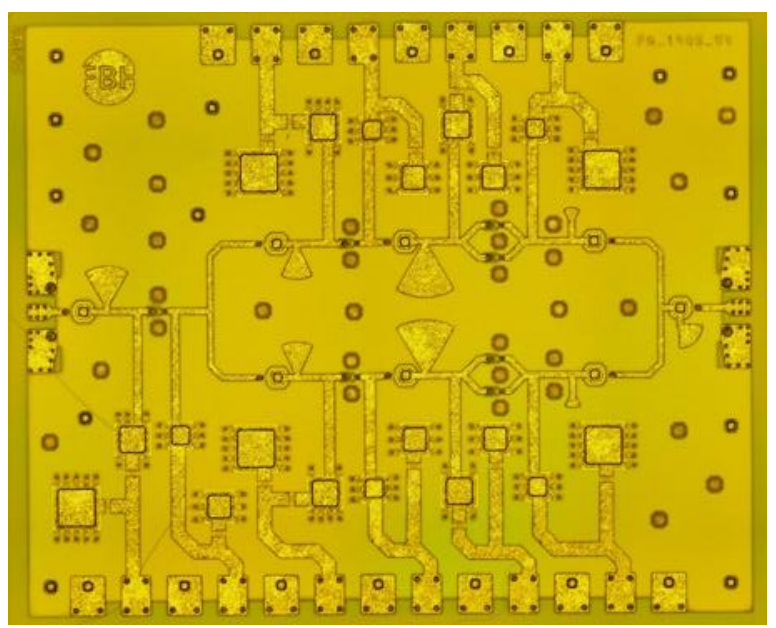

Fig.7 Microphotograph of the fabricated D-Band power amplifier

G-band Transmitter

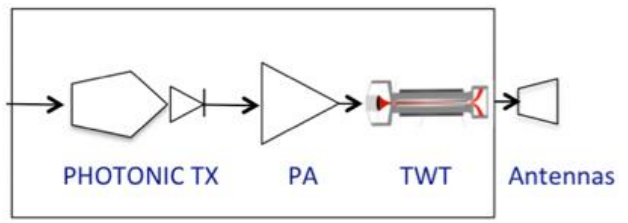

a)

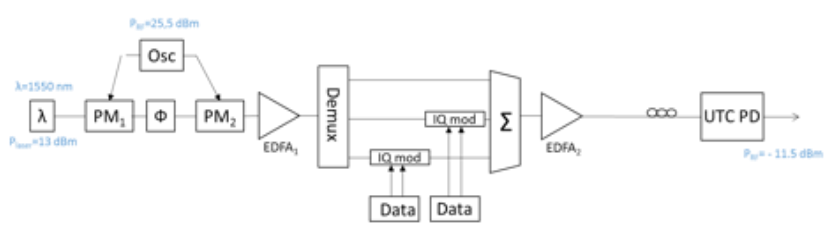

b)

Fig.8 a) G-band transmitter; b) wave form optical generator principle including electronic amplification. 
directivity antenna (38 -39 $\mathrm{dBi})$ assures an easy pointing for the range considered.

The G-band traveling wave tube will be built based on the experience of the D-band TWT. The dimensions of the radiofrequency parts will be scaled down by a factor of two in comparison to the D-band TWT. This poses a fabrication challenge and determines a lower output power. A gain of about $30 \mathrm{~dB}$ is estimated by the first simulations, with a saturated power of 1-3 W.

\section{CONCLUSIONS}

The novel wireless architecture to enable a ultracapacity layer to provide backhaul with unprecedented area capacity has been described. The system specifications were defined by accurate scenario simulation in real environment. The fabrication of the ULTRAWAVE system is in advanced status.

\section{ACKNOWLEDGMENT}

The work has received funding from the European Union's Horizon 2020 research and innovation programs under grant agreement no 762119. This work reflects only the author view's and the Commission is not responsible of any use that may be made of the information it contains

\section{REFERENCES}

[1] Ericsson Mobility Report, November 2018 on line https://www.ericsson.com/en/mobility-report

[2] Cisco Visual Networking Index: Global Mobile Data Traffic Forecast Update, 2016-2021 White Paper, Cisco Mobile VNI, 2017

[3] Dhillon, S. S. et. al, "The 2017 terahertz science and technology roadmap", J. Phys. D: Appl. Phys. 2017, 50, 043001.
[4] T. Nagatsuma et al. Advances in terahertz communications accelerated by photonics, Nature Photonics 10, 371-379 (2016)

[5] Z. Pi and F. Khan, "An introduction to millimeter-wave mobile broadband systems," IEEE Comm. Magazine, pp. 101-107, June 2011.

[6] X. Li, et al. , "Fiber-wireless- fiber link for 100-Gb/s PDM-QPSK signal transmission at W-band," IEEE Photon. Technol. Lett., Jul. 2014.

[7] T.S. Rappaport et al., "Millimeter Wave Mobile Communications for 5G Cellular: It will work!", IEEE Access, pp. 335-349, Mai 2013.

[8] J. Takeuchi, et. al. "10-Gbit/s Bi-directional wireless data transmission system using 120-GHz-band ortho-mode transducers," 2012 IEEE Radio and Wireless Symposium, 2012, pp. 63-66.A.

[9] ULTRAWAVE website [Online]. Available: http://ultrawave2020.eu

[10] R. Taori and A. Sridharan. "Point-to-multipoint in-band mmwave backhaul for 5G networks,", IEEE Communications Magazine, pp. 195201, January 2015.

[11] F. Magne, A. Ramirez, C. Paoloni, "Millimeter Wave Point to Multipoint for Affordable High Capacity Backhaul of Dense Cell Networks", IEEE Wireless Communications and Networking Conference 2018, WCNC 2018, Barcelona, Spain, April 2018.

[12] J. Shi, L. Lv, Q. Ni, H. Pervaiz and C. Paoloni, "Modeling and Analysis of Point-to-Multipoint Millimeter-Wave Backhaul Networks," in IEEE Transactions on Wireless Communications. doi: 10.1109/TWC.2018.2879109

[13] C. Paoloni, F. Magne, F. André, J. Willebois, Q. T. Le, X. Begeaud, G. Ulisse, V. Krozer, R. Letizia, M. Marilier, A. Ramirez, R. Zimmerman, "Transmission Hub and Terminals for Point to Multipoint W-band TWEETHER System", European Conference on Networks and Communications, EuCNC 2018, Ljubljana, Slovenia, June 2018.

[14] R.Basu, L. R. Billa, R. Letizia, C. Paoloni, "Design of sub-THz traveling wave tubes for high data rate long range wireless links", 2018 Semicond. Sci. Technol. 33124009

[15] R.Basu, L. R. Billa, R. Letizia, C. Paoloni, "Design of D-band Double Corrugated Waveguide TWT for Wireless Communications ", Proc. IEEE 20th Int. Vac. Electron. Conf., Busan, South Kores, April. 2019. 